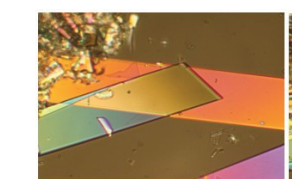

$\mathrm{Sm}_{2}\left(\mathrm{C}_{2} \mathrm{O}_{4}\right)_{3} \cdot 1 \mathrm{OH}_{2} \mathrm{O}$

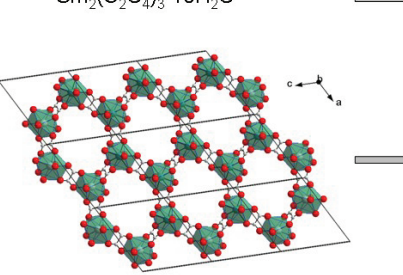

Figure 1. Changing crystal shape during dehydration of $\mathrm{Sm}_{2}\left(\mathrm{C}_{2} \mathrm{O}_{4}\right) \cdot 10 \mathrm{H}_{2} \mathrm{O}$ in relation to crystal structure. Orientation relations: $[010]_{10}^{2} / /[010]_{6},[001]_{10} / /[001]_{6},(010)_{10} / /(010)_{6}$

Keywords: dehydration reaction, structural transformation, displacive transformation, reaction-related deformation, fracture

\section{MS22-04 The solvent-dependent continuous} breathing behaviour of a wine-rack MOF

Elliot J. Carrington ${ }^{1}$, Craig McAnally², Ashleigh J. Fletcher ${ }^{2}$, Stephen P. Thompson ${ }^{3}$, Mark Warren ${ }^{3}$, Lee Brammer ${ }^{1}$

1. University of Sheffield

2. University of Strathclyde

3. Diamond Light Source

email: dtp11ejc@ @heffield.ac.uk

Metal-organic frameworks (MOFs) are a class of crystalline coordination polymers that are both highly porous and possess large internal surface areas. As a result of their structural diversity, chemical tunability and wide range of applications, these materials have gained popularity over the last couple of decades and a significant number of different framework structures are now known. Despite the large variety in the structures and in the building blocks, relatively few MOFs possess significant structural flexibility and even fewer exhibit continuous motions rather than defined phase transitions.

This talk will focus on the discovery of the novel flexibility exhibited by a previously reported wine-rack MOF. This MOF is a rare example that shows a large continuous breathing motion, which involves considerable changes in pore shape and size. These changes were not apparent from the originally published gravimetric adsorption isotherm, but have been identified by extensive crystallographic studies on the as-synthesised material. The breathing effect occurs in a single crystal-to-single crystal transformation with the change depending on the amount and type of contained solvent. These structural changes strongly affect the materials gas uptake. The selectivity has been monitored in situ using both single crystal and powder X-ray diffraction techniques

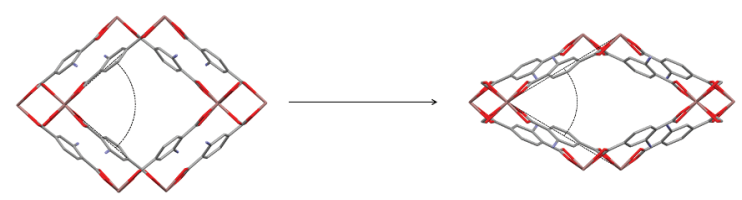

Figure 1. Representation of the changing pore shape upon loss of the as-synthesised solvent

Keywords: MOF, Flexible, Breathing 